\title{
Assainissement urbain inclusif: la délégation des services publics pour améliorer l'accès à l'assainissement dans les quartiers périphériques et non lotis de Ouagadougou
}

\author{
Jules Auguste SOW*, Hermann KAMBOU, Kuilga Marc YAMEOGO, \\ Emile NIESSOUGOU, Léon OUEDRAOGO et Halidou KOANDA
}

WaterAid Burkina Faso; E-mail: waburkinafaso@wateraid.org; Phone: + 22625374170 / + 22670447438 ; PO Box 11 BP 1211 CMS Ouagadougou 11 Burkina Faso.

*Auteur correspondant; E-mail: julessow@wateraid.org

\section{REMERCIEMENTS}

Les auteurs souhaitent remercier la délégation de l'Union Européenne dont le financement a permis la réalisation du projet.

\section{RESUME}

Renfermant $21 \%$ de la population de Ouagadougou en 2014, les quartiers périurbains non lotis avec le caractère informel et anarchique des habitats ne permettent pas une intervention classique de fourniture des services urbains de base. Près de $94 \%$ des populations n'ont pas accès à une latrines améliorée et environ 19\% défèquent à l'air libre. Cela fait des maladies hydro fécales, les principales causes de consultations médicales. Afin d'améliorer de façon inclusif l'accès à l'assainissement, WaterAid en collaboration avec la commune de Ouagadougou et l'Office Nationale de l'Eau et de l'Assainissement a initié un partenariat public -privé pour permettre aux opérateurs privés, à travers une délégation de services, de fournir dans les quartiers non lotis, les mêmes services que dans les secteurs formels. Le projet a permis de rehausser en 3 ans le taux d'accès à l'assainissement familial de $6.1 \%$ à $17 \%$. Une stratégie pilote de gestion des boues de vidange basée sur le renforcement de l'entreprenariat privé de la vidange a été initié dans 3 quartiers pilotes afin de boucler la chaine de l'assainissement. Les leçons apprises ont permis d'engager les questions d'institutionnalisation et de durabilité dans une perspective d'accès universelle en 2030.

(C) 2019 International Formulae Group. All rights reserved

Mots clés : Partenariat public-privé, quartiers non lotis, assainissement urbain, équité inclusion.

\section{Inclusive urban sanitation: The delegation of public services to improve sanitation access in the outskirts of Ouagadougou}

\begin{abstract}
With $21 \%$ of the population of Ouagadougou in 2014, the unplanned suburban neighborhoods with the informal and anarchic nature of the habitats do not allow a traditional intervention to provide basic urban services. Nearly $94 \%$ of the populations do not have access to an improved latrine and about $19 \%$ defecate in the open air. This makes fecal hydrous diseases, the main causes of medical consultations. In order to improve access to sanitation in an inclusive way, WaterAid in collaboration with the commune of Ouagadougou and the
\end{abstract}


National Office of Water and Sanitation initiated a public-private partnership to allow private operators, through a delegation of services, to provide the same services in the unplanned districts as in the formal sectors. The project has increased the rate of access to family sanitation from $6.1 \%$ to $17 \%$ in 3 years. A pilot strategy of fecal sludge management based on the reinforcement of the private draining entrepreneurship was initiated in 3 pilot districts in order to complete the chain of sanitation. The lessons learned made it possible to initiate the questions of institutionalization and sustainability in a universal access perspective in 2030 (C) 2019 International Formulae Group. All rights reserved

Keywords: public-private partnership, unplanned neighborhoods, urban sanitation, equity inclusion

\section{INTRODUCTION}

La forte croissance démographique urbaine alimentée par les migrations des populations et le phénomène de l'exode rural ont entraîné une forte extension spatiale et non contrôlée de la ville de Ouagadougou. Il en résulte une stratification spatiale de la commune en zone loties et non loties avec une forte demande en services urbains de base de plus en plus croissante. Aussi, les populations habitant les quartiers non lotis et périphériques de Ouagadougou sont rarement touchées par les projets et programmes de la commune, de l'Office National de l'Eau et de l'Assainissement (ONEA) et du gouvernement qui se limitent aux périmètres lotis ou aux villages administratifs. Le taux d'accès à l'assainissement familial dans ces quartiers était estimé à $6.1 \%$ en 2014 avec un taux de défécation à l'aire libre d'environ 19\% (Enquête WaterAid, 2014).

Ce paysage peu reluisant s'explique par l'absence de porteurs réels de la maitrise d'ouvrage communale dans les quartiers non lotis et périphériques et une implication insuffisante du secteur privé dans l'offre des services d'assainissement. Les approches et les actions jusque-là pratiquées ont atteint leurs limites pour faire face aux défis d'améliorer l'accès aux services améliorés par les populations marginalisées vivant dans ces quartiers et dont la taille est estimée à $21 \%$ de la population urbaine totale du Burkina (ENA, 2010 DGRE). Avec la lenteur et les blocages rencontrés par les projets de restructuration, il est nécessaire d'entreprendre des solutions spécifiques adaptées pour accélérer la fourniture des services dans ces quartiers défavorisées dans la perspective de l'accès universelle à l'horizon 2030.
Le projet «Assainissement durable dans les quartiers non lotis et périphériques de la commune de Ouagadougou au Burkina Faso » (PERISAN) a été élaboré en partenariat entre WaterAid, la Commune de Ouagadougou, l'Office National de l'Eau et l'Assainissement (ONEA) et l'ONG Eau Vive afin d'apporter une réponse adaptée et durable en matière de fourniture de services d'assainissement. Le projet bénéficie du financement du l'union Européenne et a pour objectifs spécifique d'améliorer l'accès aux services durables d'assainissement de 270000 personnes défavorisées de 5 quartiers non lotis et 16 secteurs périphériques, soit $67 \%$ de la population totale des quartiers non lotis et périphériques de la commune de Ouagadougou.

\section{MATERIEL ET METHODES}

La solution stratégique adoptée par le projet se base sur une approche 3 piliers schématisée sur la figure 1

Cette stratégie à trois piliers comprend :

- La capacitation des acteurs de la chaine des services d'assainissement avec la formation des animateurs communaux et privés aux techniques de mobilisations sociale pour susciter la demande services (outils ATPC, SARAR PHAST, animations grand publics); formation des artisans maçons, opérateurs de vidange des latrines, maraîchers, équipes techniques des délégataires de services afin de répondre à la demande de service sur toute la chaîne de l'assainissement.

- L'Appui à la Maitrise d'Ouvrage Communale (AMOC) avec la mise en place, formation et coaching des services techniques communaux d'assainissement 
dans toutes les mairies d'arrondissement pour le développement la planification et le suivi rapproché de toutes les activités de fourniture de services.

- La promotion de l'hygiène et marketing de l'assainissement à travers la campagne de plaidoyer, de sensibilisation et de d'éducation à l'hygiène et à l'assainissement suivant des méthodes innovantes pour éradiquer la défécation en plein air et améliorer la santé des populations.

L'approche méthodologique de fourniture des services d'assainissement développée par le projet dans les quartiers non lotis et périphériques est basée sur un partenariat public privé à travers un contrat tripartite: mairie d'arrondissement Délégataire - ONEA qui repose sur l'expérience réussie du modèle de délégation des services publics d'eau potable dans 5 quartiers non lotis de la commune (Figure 2). Le modèle s'appuie sur les opérateurs privés ayant signé un contrat de délégation avec l'ONEA et la mairie de Ouagadougou pour développer et gérer le service d'eau potable dans les quartiers non lotis aux mêmes tarifs pratiqués par l'ONEA dans les quartiers lotis. Il a consisté à élargir les activités des délégataires à la mobilisation sociale, la réalisation/réhabilitation d'ouvrages d'assainissement familial, la facilitation et l'accompagnement des communautés à accéder aux services à travers la mise en place et la gestion des boutiques d'assainissement dans les quartiers couverts par leur contrat de délégation. Les Services Techniques Communaux d'Assainissement (STCA) mis place dans les arrondissements concernés apportent leur appui aux délégataires et assurent de manière permanente le suivi de proximité, l'évaluation et la capitalisation de toutes les actions entreprises.

Dans ce schéma tripartite, les différentes parties prenantes assurent les rôles suivants :

\section{Mairie d'arrondissement}

- Assurance de la Maitrise d'ouvrage

- Elaboration du cahier des charges des délégataires
- Informations sur les normes et critères en matière d'assainissement dans la commune

- Suivi -contrôle supervision (à travers les STCA)

- Validation des plannings des délégataires

- Transmission des rapports des délégataires à l'ONEA et WaterAid

- Mobilisation sociale et sensibilisation des populations

- Réception des ouvrages

- Validation des rapports des délégataires et suivi des payements

\section{Délégataires de services publics}

\section{d'assainissement}

- Appui conseil des populations

- Gestion des boutiques d'assainissement et de la base données générale de leur zone de couverture

- Promotions de l'Hygiène, marketing des ouvrages

- Coordinations et suivi des activités et des acteurs dans la fourniture des services (mâcons, vidangeurs, ménages, fournisseurs de préfabriqués etc.)

- Gestion de la subvention aux ouvrages

- Elaboration de rapport et transmission à la commune et aux partenaires

\section{ONEA}

- Appui conseil aux différents acteurs

- Renforcement des capacités des prestataires sur la chaine des services de l'assainissement

- Suivi/contrôle de la qualité des prestations.

Dans ce positionnement stratégique, WaterAid qui n'a pas d'intervention directe dans la délivrance de services a joué son rôle d'influence sur les bonnes pratiques en améliorant la gouvernance des services et en accompagnement à la mise en œuvre de modèles et de stratégies durables, gérées de manière autonome par les institutions et acteurs locaux. Aussi les stratégies d'intervention sont élaborées en prenant en compte les impératifs de l'équité, l'inclusion et des principes du droit d'accès avec la prise en compte des personnes vulnérables et marginalisées. 
La commune de Ouagadougou à travers la Direction des Etudes, de la Planification et de l'investissement (DEPI) assure l'encadrement technique et le coaching des STCA des arrondissements ainsi que l'appui et l'ancrage institutionnel de la délégation des services publiques d'assainissement. Elle assure l'interface entre les arrondissements et la coordination du projet au niveau de WaterAid. Les sessions de renforcements des capacités et de coaching développées tout au long du projet sous l'impulsion de WaterAid devraient permettre à la commune d'assurer le leadership $\mathrm{du}$ fonctionnement du système en tant que Maitre d'Ouvrage.

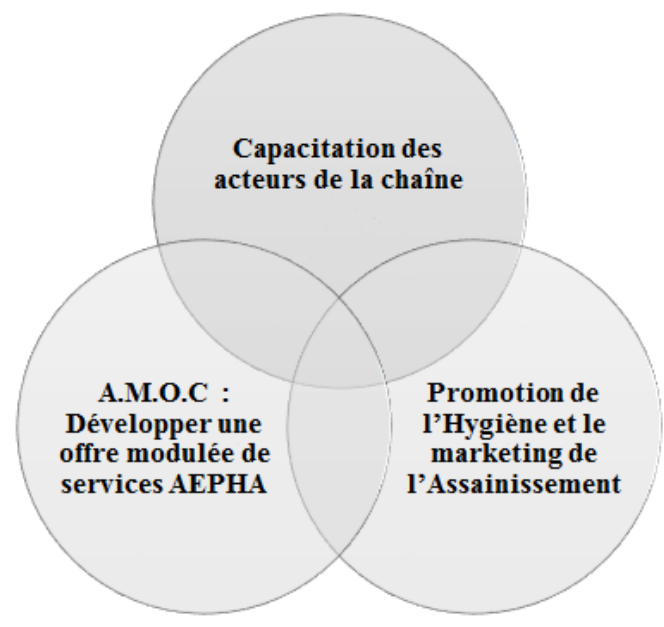

Figure 1: 3 piliers stratégiques d'intervention du PERISAN.

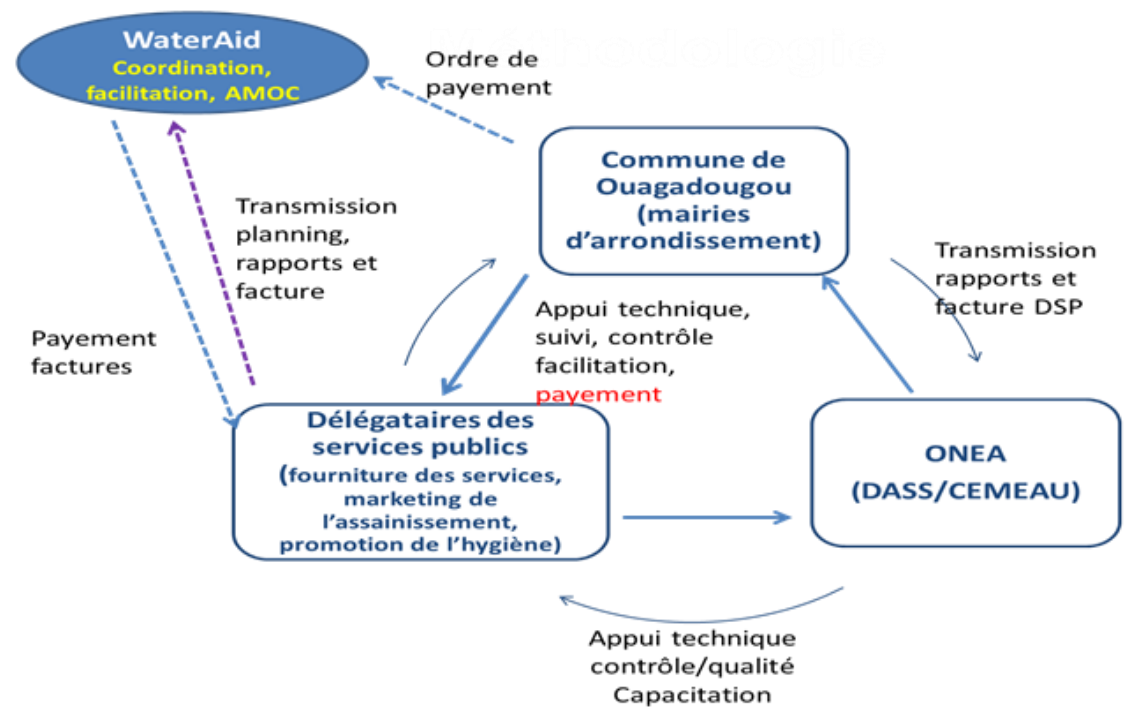

Figure 2: Schéma du contrat tripartite de Délégation de services publics. 


\section{RESULTATS}

Au bout de quatre (04) ans de mise en œuvre, les résultats du projet PERISAN sont très appréciables du point de vue de l'amélioration des services alliant les réalisations physiques à l'amélioration des capacités techniques, organisationnelles et institutionnelles des acteurs et au renforcement du secteur. Toute chose qui contribue à l'avancé vers l'accès universel à l'assainissement.

\section{Amélioration des capacités de Maitrise d'Ouvrage local}

Conformément à la stratégie du projet, trois (03) techniciens communaux d'assainissement et sept (07) chargés d'intermédiation sociale ont été recrutés par la commune de Ouagadougou avec l'appui de WaterAid sous contrat à durée indéterminée. Ces agents couvrent les 7 arrondissements du projet et chaque agent a bénéficié d'un lot de matériel composé de mobilier de bureau, de matériel informatique. L'équipe de coordination du projet en collaboration avec le Centre des Métiers de l'Eau de l'ONEA(CEMEAU), a identifié et développé/adapté les modules clés de base de la Maitrise d'Ouvrage Communale pour les services techniques et les prestataires de service notamment : la planification opérationnelle, la réalisation des ouvrages d'assainissement, le suivi technique des ouvrages, les stratégies de mobilisation sociale. Ces modules ont par la suite été adaptés au contexte du projet par le CEMEAU et toutes les formations à l'endroit des STCA et des prestataires ont été progressivement développés durant la mise en œuvre du projet. En plus des formations pratiques, les 10 agents des STCA ont bénéficié des appuis, conseils techniques de toute l'équipe de coordination $\mathrm{du}$ projet. Les séances de coaching portaient principalement sur la bonne planification et le suivi de la mise en œuvre des activités. Ce coaching des agents s'est déroulé sur toute la durée du projet au gré de nouvelles initiatives entreprises. Ainsi, le coaching continu des agents des STCA leur a permis d'organiser et d'animer les rencontres de cadrage avec les délégataires de services ainsi que les rencontres des cadres de concertation dans leurs arrondissements respectifs. Aussi, les techniciens ont participé à l'ensemble des rencontres trimestrielles organisées par WaterAid et ses partenaires durant la période d'exécution du projet. Cela a été pour eux un espace d'apprentissage et de partages d'expériences dans la planification et la mise en œuvre des activités d'assainissement.

A la clôture du projet, tous les services techniques communaux d'assainissement sont restés en service dans les arrondissements respectifs, opérationnels et totalement pris en charge (salaire et carburant) par la commune de Ouagadougou pour la promotion de l'Assainissement et l'hygiène mais aussi pour le suivi des activités relatives à l'Approvisionnement en Eau Potable des arrondissements.

\section{Amélioration de l'offre de services inclusifs d'Eau et d'Assainissement}

Pour assurer un service continu et de qualité en matière d'hygiène et d'assainissement pour les populations vivant dans les quartiers non lotis, la commune s'est appuyée sur le partenariat public - privé à travers des contrats tripartites de délégation entre l'ONEA, les mairies d'arrondissements couvrant les 5 quartiers non lotis et les 5 Délégataires que sont : (1) Adam's Commerce Distribution(ACD) (2) Atelier Construction Métallique Générale (ACMG) (3) Bureau d'Etudes et de Recherches Appliquées (BERA) (4) Entreprise Réalisation Travaux(ERT) et (5) Société Zoungrana Hamado Koudougou et Frères (SOZHAKOF).

Afin de faciliter la fourniture des services, des boutiques d'assainissement ont été mises en place par chaque délégataire et contribuent à :

- Offrir un guichet pour l'information/ l'appui- conseil des populations sur les activités du projet ;

- Présenter les prototypes des ouvrages à promouvoir ;

- Informer les ménages sur les modalités d'octroi des subventions pour la réalisation 
et réhabilitation des ouvrages d'assainissement

- Stocker et gérer les matériaux composant la subvention ;

- Enregistrer des demandes d'ouvrages;

- Mettre en relation les ménages demandeurs et les maçons ;

- Disposer d'une base des données sur les acteurs du secteur et les ouvrages réalisés.

Les équipes des délégataires ont désigné un gérant pour tenir une permanence d'information dans ces boutiques tous les jours et heures ouvrables. Le choix des sites des boutiques s'est fait en tenant compte de l'accessibilité ou la proximité d'autres quartiers. Ainsi, une boutique couvre 3 ou 4 quartiers non lotis contigus et prend en charge les besoins des ménages résidents. Une évaluation périodique des boutiques a été faite pour apprécier la qualité de services fournis et surtout formuler des recommandations en vue d'améliorer le service. La création de ces boutiques a eu une incidence positive sur les performances du projet en ce sens qu'elle a permis de rapprocher les services d'assainissement des ménages.

\section{Amélioration des capacités des opérateurs de services}

Les différents opérateurs intervenant dans la mise en œuvre du projet sur la chaine de l'Assainissement ont bénéficié de formations afin de s'approprier de la stratégie d'intervention, de maîtriser les technologies d'assainissement promues ainsi que leurs rôles et leurs responsabilités. Ainsi, les différents modules de formations dispensées ont porté sur :

- La formation sur l'approche «Assainissement Totale Portée par les Communautés (ATPC)». Elle a permis aux participants de s'imprégner des techniques de mise œuvre de l'ATPC dans les communautés avec une adaptation au cas spécifique du milieu urbain, principalement les zones non loties et les secteurs périphériques à caractère semiurbain. L'élaboration et la mise en œuvre des plans d'action en collaboration avec les techniciens communaux a permis d'effectué 30 déclenchements dans 18 quartiers non lotis et d'assurer le suivi des activités post-déclenchement pour l'obtention du statut de fin de la défécation à l'air libre (FDAL).

- La formation en «Techniques de contrôle d'ouvrages d'assainissement ", organisée par au profit des cinq (05) superviseurs des délégataires afin de les outiller à mieux contrôler les travaux de construction et assurer la qualité des ouvrages d'assainissement réalisés dans leurs quartiers d'intervention.

- La formation sur les «Techniques de sensibilisation et de marketing des ouvrages d'assainissement» avec un accent mis sur la stratégie de ciblage des personnes pauvres et vulnérables en milieu urbain au profit des 05 superviseurs des délégataires. La stratégie de formation en cascades a permis aux superviseurs de former à leur tour leur équipe d'animation respective avec l'appui de WaterAid et des STCA. Ainsi, ces équipes techniques mettent en œuvre les activités d'animations grand public, de sensibilisation, de visites à domicile et le marketing de l'assainissement dans leur quartier d'intervention avec l'appui des STCA.

- La formation sur le «E-monitoring : suivi des ouvrages d'assainissement à l'aide de smartphone », à l'endroit des 08 superviseurs (5 délégataires et 3 STCA). Elle les a permis de se familiariser avec la plateforme mWater et la technique / procédure de suivi des ouvrages d'assainissement. Ainsi, chaque opérateur a été doté de smartphones pour le suivi et le géo-référencement de tous les ouvrages réalisés avec l'appui financier du projet.

- La formation et visite d'échange sur «l'approche basée sur le marché de l'Assainissement» au profit des 05 superviseurs des délégataires et gérants des boutiques d'assainissement installées. 
- La formation sur les «Techniques de la réutilisation des sous-produits d'assainissement des eaux usées et excréta » au profit des 08 superviseurs

- La formation sur le «Suivi postdéclenchement ATPC». Elle a permis d'harmoniser les compréhensions sur les rôles des superviseurs / délégataires, sur les indicateurs à suivre et sur les fiches de suivi du post-déclenchement. Aussi, ils ont contribué à l'élaboration de plan d'action dont la mise en œuvre a été fait en collaboration avec les STCA.

- La formation sur les «Techniques de la vidange manuelle propre, la valorisation des sous-produits et l'établissement de plans d'affaire marketing ». Elle a permis aux participants de s'approprier des outils de suivi et de supervision de la vidange manuelle propre et hygiénique, d'avoir des notions sur le montage et le suivi d'un plan d'affaire marketing sur la valorisation des sous-produits de l'assainissement.

- La formation des maçons sur les «Techniques de construction des types d'ouvrages d'assainissement retenus dans Programme national d'Assainissement $»$ : elle a été dispensée au profit de 92 maçons en 03 sessions.

- La formation sur les «Techniques de construction de bidet pour l'adaptation des latrines aux personnes vulnérables ou vivant avec un handicap »: organisée au profit de 20 maçons, qui ont ensuite formé les 67 autres maçons des quartiers non lotis d'intervention du projet.

Toutes ces formations ont permis de doter les opérateurs de compétences à pouvoir fournir services adéquats selon le besoin des populations locales des quartiers non lotis.

\section{Amélioration de l'accès des populations aux services d'assainissement}

A l'issue de la signature des contrats tripartites (entre les arrondissements de la commune, l'ONEA et chaque délégataire), les délégataires ont entamé les activités de mobilisation sociale afin de stimuler les demandes de construction d'ouvrages d'assainissement. Les activités de constructions sont suivies et appuyées par les STCA et l'équipe du projet. La gestion des subventions a été faite selon le schéma du flux financiers convenu avec toutes les parties prenantes (cf. matériel et méthodes). L'octroi d'une nouvelle subvention était conditionné par la justification totale ou à hauteur d'au moins $70 \%$ de la précédente (par un rapport financier et la base de données des bénéficiaires de cette subvention).

En plus, de cette subvention classique, 160 ouvrages ont été totalement subventionnés pour les personnes vulnérables, qui avaient été identifiées par les équipes techniques des délégataires dans leurs quartiers d'intervention en collaboration avec les services de l'action sociale des arrondissements.

Avec la performance acquise par les délégataires et l'Association des Mains Unies du Sahel (AMUS) partenaires de WaterAid dans les quartiers non couverts par la délégation de services, WaterAid, a sollicité et obtenue de l'UE une augmentation du nombre de latrines à réaliser à 15880 au lieu de 15 400 initialement prévues avec les reliquats budgétaires des activités qui n'ont pas été réalisables. Finalement, 15913 ouvrages familiaux au total ont été réalisés de décembre 2014 à février 2017 par les délégataires et AMUS avec un pic de plus de 1400 ouvrages réalisés durant mars 2016 (voir figure 3). Ces réalisations ont permis à 80406 personnes d'avoir accès à des ouvrages d'assainissement adéquats dans les 30 quartiers non lotis d'intervention du projet. Tous les ouvrages réalisés ont été réceptionnés en présence des STCA, puis enregistrés dans les bases de données des prestataires qui sont disponibles chez les partenaires du projet notamment la commune de Ouagadougou, l'ONEA et WaterAid. Tous les ouvrages réalisés ont été géo référenciés et déployés sur la plateforme mWater (lien pour les détails: https://portal.mwater.co/\#/forms/dbdbac7c426 6483daf8a717a43f73ec4/visualization) 
Le tableau 1 présente la répartition du nombre d'ouvrages et de personnes soutenues pour avoir accès à l'assainissement par prestataire.

Toutes ces réalisations ont permis de rehausser le taux d'accès à l'Assainissement familial dans les quartiers non lotis de $6.1 \%$ en 2014 à environ $17 \%$ en 2017 . En plus des ouvrages familiaux et sur la base des besoins exprimés par les établissements scolaires, structures sanitaires et les lieux publics de la zone du projet, des ouvrages autonomes multipostes de type VIP ont été réalisés par l'ONEA à travers des prestataires recrutés par appel d'offre. A la date du 30 septembre 2017, fin $\mathrm{du}$ projet, 74 blocs de latrines institutionnelles ont été construits sur 39 sites (36 écoles, 02 Centres de santé et 01 marché) au profit de plus de 15000 personnes.

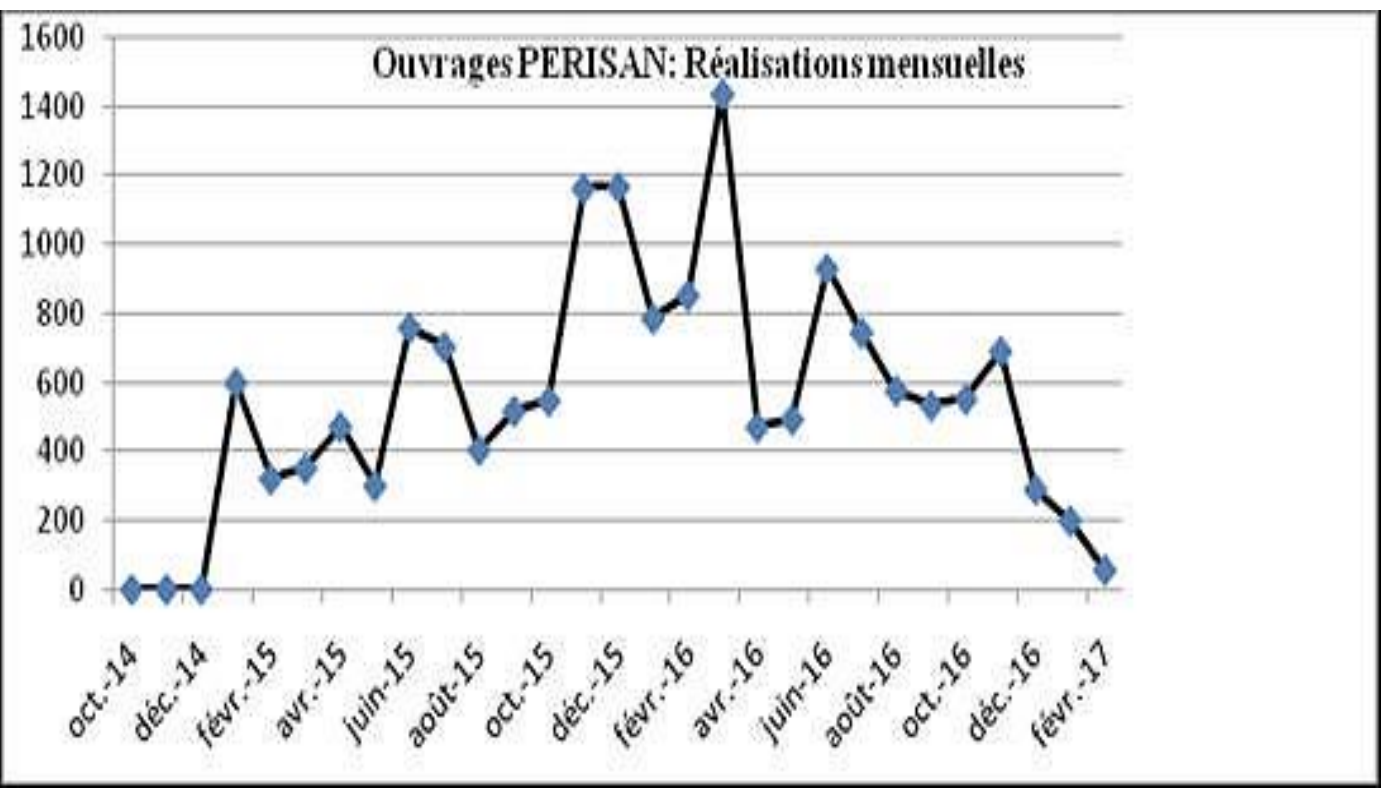

Figure 3: Réalisations mensuelles d'ouvrages d'assainissement.

Tableau 1: Réalisations par prestataires.

\begin{tabular}{lcc}
\hline Délégataires & Nombre d'ouvrages & Nombre de Bénéficiaires \\
\hline ACD & 1810 & 8627 \\
\hline ACMG & 2151 & 10490 \\
\hline BERA & 3455 & 17050 \\
\hline ERT & 1330 & 9257 \\
\hline SOZHAKOF & 2212 & 13066 \\
\hline AMUS & 4955 & 21916 \\
\hline Total & $\mathbf{1 5 9 1 3}$ & $\mathbf{8 0 4 0 6}$ \\
\hline
\end{tabular}




\section{DISCUSSION}

\section{De la pertinence du projet}

Le projet a été une opportunité de prendre en compte les quartiers périphériques non lotis dans le système d'assainissement de la ville de Ouagadougou qui regroupe $24 \%$ de la population (réf ; situation de référence, WaterAid, 2014). Le statut de non loti les exclut des services conventionnels d'eau et d'assainissement. Le projet est aligné sur les besoins et les priorités du pays et de la population cible, notamment l'accès aux services sociaux de base (santé, éducation, eau potable et assainissement, etc.) qui occupe une place éminente dans les politiques et programmes nationaux définissant les options de développement. Il contribue à l'atteinte de six (06) Objectifs du Développement Durable (ODD) : l'ODD 6 «accès à l'eau salubre et à l'assainissement», l'ODD 3 "accès à la santé ", l'ODD 10 "réduction des inégalités ", l'ODD 4 «accès à une éducation de qualité », l'ODD 9 « innovation et infrastructures» et l'ODD 11 « villes et communautés durables » (Bayili, 2017).

\section{Du respect des stratégies nationales}

Les principaux points de la stratégie du projet sont: (i) le développement d'un partenariat entre la commune de Ouagadougou et ses arrondissements, les prestataires de services publics, le secteur privé et la société civile; (ii) la capacitation des services techniques et les prestataires de service; (iii) l'élargissement des prestations des délégataires de l'ONEA sur l'hygiène et l'assainissement; (iv) l'approche par la demande; (v) la contractualisation avec les opérateurs de proximité pour la promotion de l'hygiène et de l'assainissement; (vi) l'intermédiation sociale; (vii) la subvention des ouvrages d'assainissement.

Pour la construction d'une nouvelle latrine, la réalisation de la dalle, la production de dix (10) briques pleines, l'achat d'un tuyau PVC, l'achat d'un grillage anti mouche et le paiement de la main d'œuvre du maçon (15000 Francs CFA/latrine) sont subventionnés à hauteur totale de 42650 francs CFA. Quant au ménage, il est chargé d'apporter l'eau pour la construction, de creuser la fosse de la latrine, d'apporter des briques pour la réalisation de la superstructure. Ces options sont en cohérence parfaite avec les dispositions du programme National d'Approvisionnement et Eau Potable et Assainissement (PN-AEPEA). En effet, le document d'opérationnalisation du PN-AEPA sur la base de l'étude socio-économique préconise la subvention des ouvrages d'assainissement privés jusqu'à hauteur de $90 \%$. Cette subvention porte sur les éléments de sécurité de l'ouvrage (fosse, dalle). Il en est de même pour l'approche à la demande préconisée pour marquer la responsabilisation et l'appropriation de l'ouvrage par les bénéficiaires en vue d'une utilisation effective, efficace et durable. Le but de l'intermédiation sociale est d'informer, sensibiliser pour susciter non seulement la demande mais surtout améliorer des pratiques d'hygiène dans la perspective d'un changement de comportements.

Le projet est aligné sur le plan d'actions 2016-2020 du Programme National d'Assainissement des eaux usées et excréta du fait que l'intervention vise à mettre fin à la défécation à l'air libre à travers des approches communautaires, à améliorer le taux d'accès à un service d'assainissement durable, à développer le Partenariat Public Privé (PPP) en matière d'assainissement.

\section{Des effets du projet}

Les différentes activités réalisées dans le cadre du projet ont induit des changements de comportements notables chez les ménages. Ainsi constate- t- on :

- Une évolution du taux de possession et d'utilisation des latrines: les résultats de l'enquête terrain montre que plus de $95 \%$ des ménages disposent de latrines dont 97\% d'entre eux les utilisent correctement alors que le taux d'utilisation des latrines se situait à $91 \%$ au démarrage du projet.

- Une baisse du taux de défécation à l'air libre à hauteur de 50\% par rapport au taux de défécation de la situation de référence qui est de $19 \%$

- Des latrines assez bien entretenues ;

- Près de 96\% des ménages disposent d'un dispositif de lave mains dont $98 \%$ des ménages l'utilisent (Bayili, 2017). 


\section{De la durabilité et encrage institutionnelle}

L'ancrage institutionnel des activités du projet occupe une place importante dans sa composante d'appui à la maîtrise d'ouvrage communale, sa composante de capacitation des acteurs de la chaine des services d'assainissement et dans sa composante de promotion de l'hygiène marketing de l'assainissement pour favoriser une prise en charge des problématiques majeures inhérentes aux résultats attendus. La maîtrise d'ouvrage communale a été renforcée par la mise en place des services techniques communaux d'assainissement (STCA), la capacitation des STCA, l'instauration des cadres de concertation, l'élaboration de la stratégie de promotion de l'hygiène et de l'assainissement dans les quartiers non lotis et périphériques. Si ces facteurs constituent un atout important pour le projet, certains éléments pourraient constituer des obstacles à sa durabilité. Il s'agit, entre autres, (i) du retard dans l'opérationnalisation de la chaine de valorisation des boues de vidange (ii) du retard dans le renouvellement des contrats tripartites de délégation des services entre l'ONEA, la Commune et les Délégataires, (iii) la non garantie de la poursuite de l'organisation des cadres de concertation en termes d'implication des autorités, de mobilisation des acteurs, de régularité, de financement et de mise en œuvre des recommandations issues de ces cadres.

\section{Des leçons apprises}

- La subvention est un gage d'expansion rapide de la latrinisation en milieu périurbain mais nécessite des procédures efficaces de mise à disposition des matériaux subventionnés pour éviter un coût d'opportunité aux ménages ;

- La délégation permet une certaine proximité et un suivi continu des actions d'hygiène et d'assainissement sur le terrain. Elle permet de solutionner les contraintes dues à la lourdeur administrative qui était observable dans le cadre de la collaboration ONEA-Mairie ;

- La mise en place d'un service délégué impliquant le public et le privé nécessite d'accorder suffisamment de temps dans l'établissement et la compréhension des cahiers de charge par tous et la négociation des contrats pour faciliter la mise en œuvre ;

- Il est également important dans le contexte d'un quartier non loti où les catégories de résidents sont très variées, d'accorder suffisamment de temps pour s'imprégner des besoins réels des ménages en matière de services d'assainissement afin d'avoir une mobilisation optimale des populations et d'offrir un service adapté. Au cours de la mise en œuvre de PERISAN, il est ressorti un besoin d'équipement des ménages en puisards-douches afin d'assurer une utilisation optimale des latrines ;

- La rentabilité financière des prestations d'assainissement basées sur l'intermédiation sociale et la réalisation d'ouvrages au sein des ménages n'est pas toujours évidente pour les sociétés privées. Il est donc important d'approfondir les réflexions sur des mesures publiques d'accompagnement des privés et de diversifier davantage les sources de revenus des privés en y intégrant par exemples des activités de valorisation des sous-produits et/ou la vente de l'eau ;

- Les services techniques communaux au niveau des arrondissements sont nécessaires pour faciliter la réalisation des projets de promotion d'assainissement. Ils jouent un rôle important dans la planification opérationnelle, la réalisation des ouvrages d'assainissement, le suivi technique des ouvrages, la mobilisation sociale ;

- Les formations reçues par les services techniques communaux ont été nécessaires pour faciliter les mobilisations communautaires ainsi que le suivi des réalisations d'ouvrages dans le but de contribuer au changement de comportement au sein de la population cible ;

- Les boutiques d'assainissement induisent une facilité pour la promotion et le marketing de l'assainissement dans les zones non loties et les quartiers périphériques des villes

- La gestion et la valorisation écologique et sanitaire des boues de vidange est un 
maillon indispensable pour boucler la chaine des services de l'assainissement ;

- Les aménagements urbains et péri-urbains doivent prévoir des sites de dépotage et de traitement des boues de vidange afin d'éviter les dépotages anarchiques avec tous les risques sanitaires et environnementaux induits ;

- La programmation des activités de réalisation des ouvrages d'assainissement et de promotion de l'assainissement familial doit être en phase avec la saison pluvieuse. La réalisation des ouvrages est rendue difficile durant la saison pluvieuse ;

- Les activités d'IEC relatives à l'hygiène et à l'assainissement suscitent une plus grande adhésion et contribuent à un éveil de conscience des populations.

\section{Conclusion}

La communauté internationale a fixé à 2030 la date butoir pour tout le monde, partout dans le monde, pour avoir accès à l'eau potable et à l'assainissement. Cet objectif a été coopté dans de nombreuses politiques nationales de la sous-région africaine. Cependant, il est évident que les politiques ne sont pas toujours suivies de stratégies d'expansion des services urbains de base aux populations vivant dans les banlieues défavorables des grandes villes. Le partenariat public-privé avec la Délégation de services offre une alternative crédible pour un approvisionnement durable et adapté en eau et assainissement vers l'accès universel. Cependant, son efficacité exige un engagement fort de toutes les parties prenantes ainsi qu'un soutien politique au plus haut niveau

\section{CONFLITS D'INTERETS}

Les auteurs déclarent n'avoir aucun conflit d'intérêts.

\section{CONTRIBUTIONS DES AUTEURS}

JAS est le coordonnateur du projet ; il a assuré la synthèse documentaire et l'élaboration du présent manuscrit. HK, responsable du suivi évaluation, a assuré la coordination des activités d'évaluation et d'établissement des rapports périodiques du projet qui ont servi à alimenter le présent manuscrit. KMY, EN et LO, assistants techniques, ont contribué à la mise en œuvre technique du projet et à la production des rapports finaux. HK, Directeur Pays de WaterAid Burkina, a assuré la supervision de l'ensemble du projet et l'assurance qualité des documents et du présent manuscrit.

\section{REMERCIEMENTS}

Les auteurs souhaitent remercier :

- Tous le personnel de WaterAid Burkina de WaterAid Région Afrique de l'Ouest et Londres pour leur appui technique et soutien constant aux réflexions, les appuis conseils et soutien permanent à la réalisation des actions ainsi que

- Tous les partenaires du projet PERISAN: ONEA, EAU VIVE, COMMUNE DE OUAGADOUGOU.

\section{REFERENCES}

Bayili P. 2017. Rapport d'évaluation finale du projet PERISAN, $59 \mathrm{p}$.

DGRE. 2011. Enquête Nationale sur l'Assainissement au Burkina Faso.

PériSan. 2015. Situation de référence en Assainissement, Approvisionnement en Eau Potable et Hygiène dans les quartiers non lotis et périphériques de la commune de Ouagadougou, rapport final, novembre 2015, $126 \mathrm{p}$.

PériSan. 2014. Monographie de l'Arrondissement 3 en matière d'accès aux services d'assainissement, d'hygiène et d'eau potable, $5 \mathrm{p}$.

Water Aid. 2016. Evaluation interne à miparcours du projet d'assainissement durable dans les quartiers non lotis et périphériques de la commune de Ouagadougou au Burkina Faso, 25 p.

WaterAid. 2017. Rapport narratif final du projet Assainissement durable dans les quartiers non lotis et périphériques de la commune de Ouagadougou au Burkina Faso (PériSan), 53 p.

WATERAID, 2015 «Rapport intermédiaire an $3 \mathrm{du}$ projet PERISAN », WATERAID, oct. 2015. 